\title{
Asymmetric Synthesis of Griffipavixanthone Employing a Chiral Phosphoric Acid-Catalyzed Cycloaddition
}

\author{
Michael J. Smith, Kyle D. Reichl, Randolph A. Escobar, Thomas J. Heavey, David F. Coker, \\ Scott E. Schaus*, and Jr. John A. Porco* \\ Department of Chemistry and Center for Molecular Discovery (BU-CMD), Boston University, 590 \\ Commonwealth Avenue, Boston, Massachusetts 02215, United States
}

\begin{abstract}
Asymmetric synthesis of the biologically active xanthone dimer griffipavixanthone is reported along with its absolute stereochemistry determination. Synthesis of the natural product is accomplished via dimerization of a $p$-quinone methide using a chiral phosphoric acid catalyst to afford a protected precursor in excellent diastereo- and enantioselectivity. Mechanistic studies, including an unbiased computational investigation of chiral ion-pairs using parallel tempering, were performed in order to probe the mode of asymmetric induction.
\end{abstract}

The dimeric natural product (+)-griffipavixanthone $(\mathbf{1})^{1}$ has become a desirable synthetic target due to its interesting framework and anticancer properties. Compound (+)-(1) has been shown to increase intracellular reactive oxygen species (ROS) and cleave caspase-3, thereby inducing apoptosis in lung cancer cells. ${ }^{2}$ Recent studies have shown that (+)-(1) is a B-Raf and C-Raf inhibitor in esophageal cancer cell lines with comparable effects to FDAapproved drugs. ${ }^{3}$

We have previously reported the biomimetic synthesis of $( \pm)-\mathbf{1}$ wherein treatment of $p$ quinone methide ( $p$-QM) 2 with Lewis or Brønsted acids triggered an isomerization-cycloaddition-cyclization cascade affording the polycyclic core (Scheme 1). The reaction sequence began with acid-mediated isomerization of the vinyl $p$-QM 2 to 1,3-diene 3 . Cationic, stepwise [4+2] cycloaddition of $\mathbf{2}$ and $\mathbf{3}$ afforded the anti-cycloadduct $\mathbf{4}$ and syncycloadduct $\mathbf{5}$, the latter which underwent intramolecular arylation to afford griffipavixanthone tetramethyl ether $\mathbf{6}^{4}$

The complexity and unique nature of this cationic cascade prompted our interest to undertake an asymmetric synthesis. In this regard, we considered that enantioselectivity could be introduced via chiral ion-pairing catalysis. ${ }^{5-7}$ Asymmetric [4+2] cycloadditions are

\footnotetext{
*Corresponding Authors: porco@bu.edu, seschaus@bu.edu. ASSOCIATED CONTENT

Supporting Information

The Supporting Information is available free of charge on the ACS Publications website at DOI: 10.1021/jacs.8b12520.

Video clip from the parallel tempering (PT) simulation of TS-1 and CPA F (AVI)

Experimental procedures, computational data, and characterization data for new compounds (PDF)

The authors declare no competing financial interest.
} 
mediated by both Brønsted and Lewis acids. ${ }^{8} 9$ Scheme 2 depicts select literature reports of chiral Brønsted acid-mediated asymmetric reactions involving $p$-QMs. Sun and co-workers have reported CPA-mediated, enantioselective 1,6-arylations of $p$-QMs (Scheme 2a). ${ }^{10,11} \mathrm{In}$ addition, $\mathrm{Li}$ and co-workers have demonstrated asymmetric $a$ - alkylations to $p$-QMs generating two contiguous stereocenters (Scheme $2 b$ ). ${ }^{12}$ There is a single report of a diastereoselective, CPA-mediated intramolecular [4+2] cycloaddition. ${ }^{13}$ These studies further prompted our interest to develop an intermolecular, asymmetric [4+2] cycloaddition of a $p$-QM (Scheme 2c). Herein, we (I) describe a CPA-catalyzed asymmetric synthesis of $(+)-\mathbf{1},(2)$ demonstrate catalyst- dependent diastereoselectivity, (3) assign absolute stereochemical configuration to (+)-1, and (4) provide computational insight for the asymmetric induction observed using parallel-tempering (PT) simulation methods.

Yamamoto and co-workers have demonstrated that chiral Brønsted acid-mediated cycloadditions require specific $\mathrm{p} K_{\mathrm{a}}$ 's as dictated by the substrate. ${ }^{14}$ With this in mind, our investigation began by surveying chiral Brønsted acids with varying $\mathrm{p} K_{a}$ values including CPAs, imidodiphosphoric acids (IDPs), and $N$-triflyl phosphorimides. ${ }^{14-17}$ While the latter two catalyst classes chiefly led to isomerization of $p$-QM 2 to diene 3 , use of CPAs led to production of cycloadducts 4-6. A study of electronically variable CPAs was performed (Table 1). We found that treatment of $\mathbf{2}$ with CPA A ${ }^{18}$ (entry 1) generated dimer $\mathbf{6}$ in high enantioselectivity along with products $3-5$. Electron rich CPAs $\mathbf{B}$ and $\mathbf{C}^{19}$ lowered the reactivity for cycloadducts (entries 2 and 3). With the most promising result observed using $-\mathrm{CF}_{3}$ catalyst $(\mathbf{A})$, we expanded our assessment of electron-deficient, aryl-substituted CPAs and found that the highly electron-deficient Yamamoto catalyst (CPA D $)^{20}$ favored the anti-cycloadduct 4 (entry 4). However, the moderately electron deficient CPA $\mathbf{E}^{21}$ afforded several products, while still maintaining high enantioselectivity for dimer $\mathbf{6}$ (entry 5). Interestingly, use of CPA $\mathbf{F}^{19}$ bearing the strongly electron-withdrawing nitro $\left(\mathrm{NO}_{2}\right)$ substituent provided high diastereo- and enantioselectivity (entry 6). At elevated temperatures (entry 7), the reaction was driven to completion affording (+)-6 in $72 \%$ yield and $99 \%$ ee.

To determine the absolute stereochemistry of $\mathbf{1}$ prepared using CPA $\mathbf{F}$, global demethylation of (+)-6 using our previously reported conditions ${ }^{4}$ was performed. By measuring the optical rotation of $\mathbf{1}$, we found that $(R)$-CPA $\mathbf{F}$ provided the natural (+)-enantiomer of $\mathbf{6}$ (Figure 1a). To elucidate the absolute stereochemistry of (+)-1, an electronic circular dichroism (ECD) spectrum was obtained. Comparison with the theoretical ECD spectrum (CAM-B3LYP/ def2-TZVP/ PCM (MeOH)//CAM-B3LYP/def2-SVP/PCM (MeOH) indicates that (+)-1 has $8 R, 8 \mathrm{a} R, 12 S$ stereochemistry (Figure $1 \mathrm{~b}){ }^{22}$

At this juncture, we were also interested to determine the enantioselectivity of the anticycloadduct, the only dimeric product formed upon treatment of $\mathbf{2}$ with CPA D. Upon isolation of the dimeric $p$-QM cycloadduct 4 , heterogeneous reduction with $\mathrm{H}_{2} / \mathrm{Pd} / \mathrm{C}$ afforded cycloadduct 7 in 33\% yield (2 steps) and $71 \%$ ee (Scheme 3 ). The absolute stereochemistry of (+)-7 was determined using ECD analysis. ${ }^{22}$

We also used in situ ${ }^{1} \mathrm{H}$ NMR analysis to monitor reactions comparing both TFA and CPA $\mathbf{F}$ as catalysts. Using TFA as a catalyst, production of the anti-cycloadduct $\mathbf{4}$ appears to be 
more favorable than the syn-cycloadduct 5 (Figure 2a). Remarkably, CPA $\mathbf{F}$ shows reversal of the TFA-catalyzed preference for anti-cycloadduct 4, instead showing initial formation of the syn-cycloadduct $\mathbf{5}$ (Figure 2b). Presumably, CPA catalyst $\mathbf{F}$ reduces the energetic barrier to the syn-cycloadduct 5 (vide infra). ${ }^{1} \mathrm{H}$ NMR data (Figure 2) also indicated formation of diene 3 in situ. To probe the potential for decomposition or dimerization, diene $\mathbf{3}$ was subjected to the standard conditions; in the event, 6 was isolated in $69 \%$ yield and $96 \%$ ee. ${ }^{22}$

As we identified catalyst diastereoselectivity, we were also interested to probe the point at which enantioselectivity was introduced. Treatment of $( \pm)-\mathbf{5}$ with CPA $\mathbf{F}$ resulted in the formation of (+)-6 in 78\% ee (Scheme 4a). In a control experiment, $( \pm)-4$ was subjected to the same conditions, affording (+)-6 in $99 \%$ ee. These results indicate that reorganization to the syn-cycloadduct 5 is the enantiodetermining step. To further understand enantioinduction, the reaction was stopped after $3 \mathrm{~min}$ and the $s y n$-cycloadduct 5 was purified and isolated in 53\% yield and 99\% ee (Scheme 4b). This reaffirmed that the formation of the syn-cyclohexene moiety is the enantiodetermining step (cf. 5, Scheme 1). In addition, we performed kinetics studies using React IR analysis. The data obtained is consistent with a first-order reaction with respect to catalyst. ${ }^{22}$

Previous literature has shown that CPAs promote reactivity through the Brønsted acidic site, the Lewis basic site, or both. This interaction generates transient diastereomeric ion-pairs, one of which is more favorable. ${ }^{23}$ Notably, density functional theory (DFT) calculations have been used to elucidate the primary phosphate interaction and the secondary stereoelectronic interactions imparting enantioselectivity. ${ }^{24-27}$

To investigate the mode of enantioinduction, we used a computational methodology that has not been employed as a tool in organic synthesis. As we were unsure of the mode of asymmetric induction leading to $\mathbf{5},{ }^{4}$ we used an enhanced-sampling ${ }^{28}$ computational method to explore configurations with CPA $\mathbf{F}$ in an unbiased manner. Parallel tempering (PT) simulation ${ }^{29}$ were conducted using a molecular-mechanics force field. ${ }^{30}$ In these calculations, multiple independent simulations are run in parallel at a range of temperatures; this enables frequent crossing of energetic barriers, thereby fully sampling the thermal equilibrium distribution. Applications of PT methodology primarily include biomolecular dynamics to further understand protein structure. ${ }^{31-35}$ A representative video from a simulation involving the preferred enantiomeric TS and CPA $\mathbf{F}$ is provided. ${ }^{36}$

The ensembles of configurations of the transition-states (TS-1 = major, ent $\mathbf{- T S}-\mathbf{1}=$ minor) and CPA $\mathbf{F}$ generated by these PT simulations contain many structures exhibiting simultaneous double hydrogen-bonds (DHBs), where the catalyst acts as a Brønsted acid and a Lewis base. Representative structures of TS-1-CPA $\mathbf{F}$ and ent-TS-1-CPA $\mathbf{F}$ complexes sampled during these simulations are presented in Figure 3a,b, respectively. Hydrogen bonding interactions between two phenols on both xanthone fragments ${ }^{37}$ with the phosphate moiety of the CPA provide a template for long-range chirality transfer.

Consequently, histograms of the probability distributions were used to compute free energy surfaces of hydrogen bonding distances for both TS-1 and ent-TS-1 (Figure 3c,d, respectively). As expected, the lowest free energy structures are observed when CPA $\mathbf{F}$ is 
doubly hydrogen bound. The simulation of the TS-1-CPA F reveals evidence of significantly more complexes in which TS-1 hydrogen bonds to a single phosphate oxygen ${ }^{22}$ (Figure 3c) compared to ent-TS-1 (Figure 3d). The increased frequency of these intermediate SHB structures results from the enhanced flexibility of TS-1-CPA F compared to the ent-TS-1-CPA F. The intermolecular dihedral distribution in Figure 4a shows that the conformational space for TS-1 relative to CPA F permits free rotation of the bis-xanthone transition state (cf. Figure $4 \mathrm{c}$ for atoms involved). In Figure 4b, insurmountable barriers hinder relative rotation of ent-TS-1-CPA F, dramatically reducing conformational space (cf. Figure $3 b$ ) in the presence of CPA F. Figure 4c depicts the rotational freedom of TS-1 around the catalyst while maintaining long-range chirality transfer. These findings suggest that enantioinduction is derived, in part entropically, by reducing the configuration space available to ent-TS-1. Thus, TS-1-CPA F can interact by a SHB or DHB while the minor can only bind to the transition state in a DHB configuration.

In addition, $\pi$-stacking interactions with the catalyst were also found to be prominent. ${ }^{22}$ Previous studies have observed electrostatic donor-acceptor aromatic stacking effects wherein nitrophenyl substituents are among the strongest acceptors. ${ }^{38}$ These strong $\pi$ interactions play an important role in stabilizing the TS-1-CPA F complex, driving enantioselectivity.

The CPA $\mathbf{F}$ catalyst also promotes enthalpic stabilization of the major TS-1 enantiomer. A random selection of complexes from PT simulations were DFT-optimized (B3LYP/6$31 \mathrm{G}(\mathrm{d}) / \mathrm{IEF}-\mathrm{PCM}\left[\mathrm{CH}_{2} \mathrm{Cl}_{2}\right]$ to obtain energies for the catalyst-transition state complexes in the presence of a polarizable continuum model (PCM) solvent. On average, the major TS-1CPA F complex is energetically more favorable than its minor enantiomeric counterpart by nearly $7 \mathrm{kcal} / \mathrm{mol}^{22}$

On the basis of this model, we were interested in developing a catalyst that may perturb enantioinduction by steric interactions (Scheme 5). Use of the sterically modified CPA G permitted the formation of dimer 6 , albeit in lower yield and ee $(23 \%$ and $42 \%$, respectively). Erosion of enantioselectivity may be explained by steric interference between the substrate and the methyl groups of CPA G. To rationalize this outcome, PT simulations were also conducted. Interestingly, the major enantiomeric transition state (TS-1-CPA G) deviated from that of CPA F. In the TS-1-CPA F system, the nitro aryl groups are stacking with the xanthone moieties (Figure 5a). However, the added sterics of CPA G prevent $\pi$ stacking, resulting in the nitro aryl groups being forced out of plane with the xanthone (Figure 5b). DFT assessment revealed only a $1.5 \mathrm{kcal} / \mathrm{mol}$ energy difference between transition states, providing computational support for the erosion of enantioselectivity. The calculated free energy surfaces and energy distributions for the TS-1-CPA G system are provided in the Supporting Information. ${ }^{22}$

In summary, we have reported asymmetric syntheses and absolute stereochemistry assignment of (+)- and (-)-griffipavixanthone, a biologically active xanthone dimer. The key cascade reaction utilizes a chiral phosphoric acid (CPA)- catalyzed cycloaddition of a paraquinone methide ( $p$-QM) monomer in which a specific aryl nitro-substituted CPA catalyst provides high diastereo- and enantioselectivity. In addition, we have interrogated the 
mechanism and mode of enantioinduction through catalyst electronic effects, in situ ${ }^{1} \mathrm{H}$ NMR reaction profiles, kinetic studies, and computational studies using parallel tempering (PT) simulations to broadly sample chiral ion pair complexes. Further studies on the synthesis of xanthone dimers as well as applications of parallel tempering (PT) to address problems in organic synthesis are currently in progress and will be reported in due course.

\section{Supplementary Material}

Refer to Web version on PubMed Central for supplementary material.

\section{ACKNOWLEDGMENTS}

We thank the National Institutes of Health (NIH) (R35 GM- 118173 to J.A.P., Jr.) and Boston University (BU) for financial support. S.E.S. and R.A.E. acknowledge the NIH for support (R01 GM-078240). K.D.R is supported by a postdoctoral fellowship (PF-16-235-01-CDD) from the American Cancer Society. NMR (CHE-0619339) and MS (CHE-0443618) facilities at BU are supported by the National Science Foundation (NSF). We also thank the NSF (CHE-1665367 to D.F.C.) for funding. Work at the BU-CMD is supported by the NIH (R24 GM-111625). We acknowledge Prof. Richard Johnson and Dr. Sharon Song (UNH) for their computational investigations in the synthesis of $( \pm)-\mathbf{1}$. We also thank Prof. Benjamin List (MPI) for kindly providing catalysts for our initial screen.

\section{REFERENCES}

(1). Xu YJ; Cao SG; Wu XH; Lai YH; Tan BHK; Pereira JT; Goh SH; Venkatraman G; Harrison LJ; Sim KY Griffipavixanthone, a Novel Cytotoxic Bixanthone from Garcinia Griffithii and G. Pavifolia. Tetrahedron Lett. 1998, 39, 9103-9106.

(2). Shi JM; Huang HJ; Qiu SX; Feng SX; Li XE Griffipavixanthone from Garcinia Oblongifolia Champ Induces Cell Apoptosis in Human Non-Small-Cell Lung Cancer H520 Cells in Vitro. Molecules 2014, 19, 1422-1431. [PubMed: 24473206]

(3). Ding Z; Lao Y; Zhang H; Fu W; Zhu L; Tan H; Xu H Griffipavixanthone, a Dimeric Xanthone Extracted from Edible Plants, Inhibits Tumor Metastasis and Proliferation via Downregulation of the RAF Pathway in Esophageal Cancer. Oncotarget 2016, 7, 1826-1837. [PubMed: 26646323]

(4). Reichl KD; Smith MJ; Song MK; Johnson RP; Porco JA Jr. Biomimetic Total Synthesis of ( \pm )Griffipavixanthone via a Cationic Cycloaddition-Cyclization Cascade. J. Am. Chem. Soc. 2017, 139, 14053-14056. [PubMed: 28942643]

(5). Brak K; Jacobsen EN Asymmetric Ion-Pairing Catalysis. Angew. Chem.j Int. Ed. 2013, 52, 534561.

(6). Held FE; Grau D; Tsogoeva SB Enantioselective Cycloaddition Reactions Catalyzed by BINOLDerived Phosphoric Acids and N-Triflyl Phosphoramides: Recent Advances. Molecules 2015,20, 16103-16126. [PubMed: 26404222]

(7). Maji R; Mallojjala SC; Wheeler SE Chiral Phosphoric Acid Catalysis: From Numbers to Insights. Chem. Soc. Rev. 2018, 47, 1142-1158. [PubMed: 29355873]

(8). Ryu DH; Corey EJ Triflimide Activation of a Chiral Oxazaborolidine Leads to a More General Catalytic System for Enantioselective Diels-Alder Addition. J. Am. Chem. Soc. 2003, 125, 63886390. [PubMed: 12785777]

(9). Futatsugi K; Yamamoto H Oxazaborolidine-Derived Lewis Acid Assisted Lewis Acid as a Moisture-Tolerant Catalyst for Enantioselective Diels-Alder Reactions. Angew. Chem., Int. Ed. 2005, 44, 1484-1487.

(10). Wang Z; Wong YF; Sun J Catalytic Asymmetric 1,6- Conjugate Addition of Para-Quinone Methides: Formation of All Carbon Quaternary Stereocenters. Angew. Chem.j Int. Ed. 2015, 54, 13711-13714.

(11). Wong YF; Wang Z; Sun J Chiral Phosphoric Acid Catalyzed Asymmetric Addition of Naphthols to Para-Quinone Methides. Org. Biomol Chem. 2016, 14, 5751-5754. 
(12). Li W; Xu X; Liu Y; Gao H; Cheng Y; Li P Enantioselective Organocatalytic 1,6-Addition of Azlactones to Para-Quinone Methides: An Access to $a, a$-Disubstituted and $\beta, \beta$ - Diaryl- $\alpha$ Amino Acid Esters. Org. Lett. 2018, 20, 1142-1145. [PubMed: 29363977]

(13). Wang LL; Candito D; Dräger G; Herrmann J; Muller R; Kirschning A Harnessing a p-Quinone Methide Intermediate in the Biomimetic Total Synthesis of the Highly Active Antibiotic 20Deoxy- Elansolid B1. Chem. - Eur. J. 2017, 23, 5291-5298. [PubMed: 28195370]

(14). Nakashima D; Yamamoto H Design of Chiral N-Triflyl Phosphoramide as a Strong Chiral Brønsted Acid and Its Application to Asymmetric Diels-Alder Reaction. J. Am. Chem. Soc. 2006, 128, 9626-9627. [PubMed: 16866505]

(15). Jiao P; Nakashima D; Yamamoto H Enantioselective 1,3- Dipolar Cycloaddition of Nitrones with Ethyl Vinyl Ether: The Difference between Brønsted and Lewis Acid Catalysis. Angew. Chem. 2008, 120, 2445-2447.

(16). Rueping M; Uria U; Lin MY; Atodiresei I Chiral Organic Contact Ion Pairs in Metal-Free Catalytic Asymmetric Allylic Substitutions. J. Am. Chem. Soc. 2011, 133, 3732-3735. [PubMed: 21355548]

(17). Xie Y; List B Catalytic Asymmetric Intramolecular [4 + 2] Cycloaddition of In Situ Generated Ortho-Quinone Methides. Angew. Chem.j Int. Ed. 2017, 56, 4936-4940.

(18). Akiyama T; Morita H; Itoh J; Fuchibe K Chiral Brønsted Acid Catalyzed Enantioselective Hydrophosphonylation of Imines: Asymmetric Synthesis of a-Amino Phosphonates. Org. Lett. 2005, 7, 2583-2585. [PubMed: 15957896]

(19). Akiyama T; Itoh J; Yokota K; Fuchibe K Enantioselective Mannich-Type Reaction Catalyzed by a Chiral Brønsted Acid. Angew. Chem.j Int. Ed. 2004, 43, 1566-1568.

(20). Zhou F; Yamamoto H A Powerful Chiral Phosphoric Acid Catalyst for Enantioselective Mukaiyama — Mannich Reactions. Angew. Chem., Int. Ed. 2016, 55, 8970-8974.

(21). Lebée C; Kataja AO; Blanchard F; Masson G Formal Asymmetric Organocatalytic [3 + 2] Cyclization between Enecarba- mates and 3-Indolylmethanols: Rapid Access to 3Aminocyclopenta- [b]indoles. Chem. - Eur. J. 2015, 21, 8399-8402. [PubMed: 25892287]

(22). See the Supporting Information for complete experimental details.

(23). Merad J; Lalli C; Bernadat G; Maury J; Masson G Enantioselective Brønsted Acid Catalysis as a Tool for the Synthesis of Natural Products and Pharmaceuticals. Chem. - Eur. J. 2018, 24, 39253943. [PubMed: 28981209]

(24). Champagne PA; Houk KN Origins of Selectivity and General Model for Chiral Phosphoric AcidCatalyzed Oxetane Desymmetrizations. J. Am. Chem. Soc. 2016, 138, 12356-12359. [PubMed: 27629045]

(25). Reid JP; Simón L; Goodman JM A Practical Guide for Predicting the Stereochemistry of Bifunctional Phosphoric Acid Catalyzed Reactions of Imines. Acc. Chem. Res. 2016, 49, 10291041. [PubMed: 27128106]

(26). Dong N; Zhang ZP; Xue XS; Li X; Cheng JP Phosphoric Acid Catalyzed Asymmetric 1,6Conjugate Addition of Thioacetic Acid to Para-Quinone Methides. Angew. Chem., Int. Ed.2016 55, 1460-1464.

(27). Reid JP; Goodman JM Selecting Chiral BINOL-Derived Phosphoric Acid Catalysts: General Model To Identify Steric Features Essential for Enantioselectivity. Chem. - Eur. J. 2017, 23, 14248-14260. [PubMed: 28771900]

(28). For a recent example of enhanced-sampling (molecular dynamics) computational analysis of a peptide catalyst, see: Yan XC; Metrano AJ; Robertson MJ; Abascal NC; Tirado-Rives J; Miller SJ; Jorgensen WL Molecular Dynamics Simulations of a Conformationally Mobile PeptideBased Catalyst for Atroposelective Bromination. ACS Catal 2018, 8, 9968-9979. [PubMed: 30687577]

(29). Sugita Y; Okamoto Y Replica-Exchange Molecular Dynamics Method for Protein Folding. Chem. Phys. Lett. 1999, 314, 141-151.

(30). Wang J; Wolf RM; Caldwell JW; Kollman PA; Case DA Development and Testing of a General Amber Force Field. J. Comput. Chem. 2004, 25, 1157-1174. [PubMed: 15116359] 
(31). Bussi G; Gervasio FL; Laio A; Parrinello M Free-Energy Landscape for $\beta$ Hairpin Folding from Combined Parallel Tempering and Metadynamics. J. Am. Chem. Soc. 2006, 128, 13435-13441. [PubMed: 17031956]

(32). Coskuner O; Wise-Scira O Structures and Free Energy Landscapes of the A53T Mutant-Type aSynuclein Protein and Impact of A53T Mutation on the Structures of the Wild-Type a- Synuclein Protein with Dynamics. ACS Chem. Neurosci. 2013, 4, 1101-1113. [PubMed: 23607785]

(33). Luitz M; Bomblies R; Ostermeir K; Zacharias M Exploring Biomolecular Dynamics and Interactions Using Advanced Sampling Methods. J. Phys.: Condens. Matter 2015, 27, 323101. [PubMed: 26194626]

(34). Zhao W; Xu Z; Cui Q; Sahai N Predicting the Structure- Activity Relationship of Hydroxyapatite-Binding Peptides by Enhanced-Sampling Molecular Simulation. Langmuir 2016, 32, 7009-7022. [PubMed: 27329793]

(35). For a review of Parallel Tempering including non-protein applications, see: Earl DJ; Deem MW Parallel Tempering: Theory, Applications, and New Perspectives. Phys. Chem. Chem. Phys. 2005, 7, 3910-3916. [PubMed: 19810318]

(36). A video clip from the parallel tempering (PT) simulation of TS- 1 and CPA F can be found in.avi format as a supplemental file. The clip includes 1500 sequential frames of the 1 million obtained from the simulation.

(37). Ito K; Nishiki M; Ohba Y Interaction between Biphenols and Anions: Selective Receptor for Dihydrogenphosphate. Chem. Pharm. Bull. 2005, 53, 1352-1354. [PubMed: 16205002]

(38). Cockroft SL; Hunter CA; Lawson KR; Perkins J; Urch CJ Electrostatic Control of Aromatic Stacking Interactions. J. Am. Chem. Soc. 2005, 127, 8594-8595. [PubMed: 15954755] 

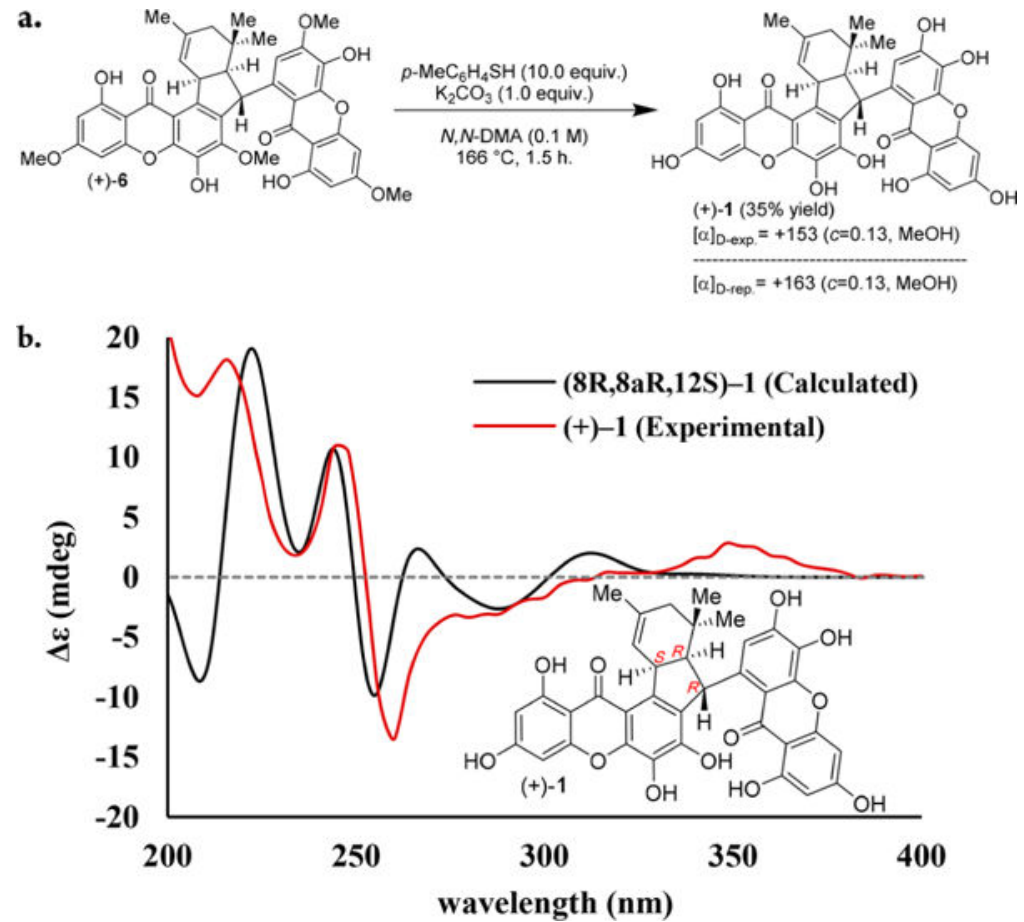

Figure 1.

Absolute stereochemical determination of (+)-1. (a) Synthesis of (+)-1. (b) Comparison of experimental and computational ECD spectra for (+)-1. 
a.

$t=0 \min$
a.

i

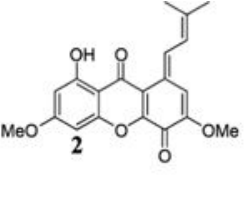

$\int$

$t=2 \min$

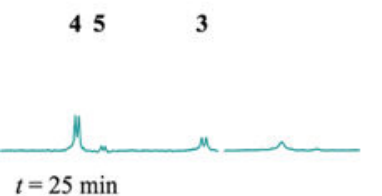

$t=25 \mathrm{~min}$
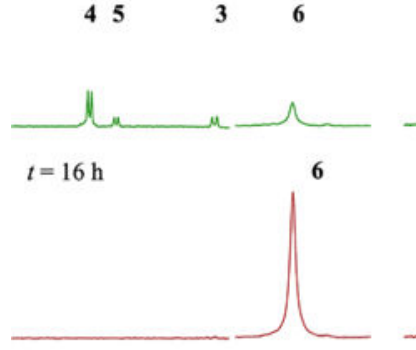

$\begin{array}{llllll}9.5 & 9.0 & 8.5 & 8.0 & 0.7 & 0.6\end{array}$

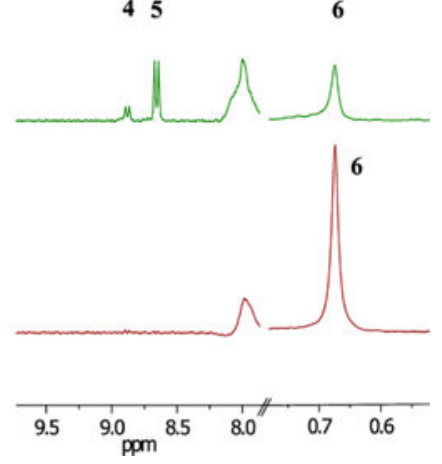

b.
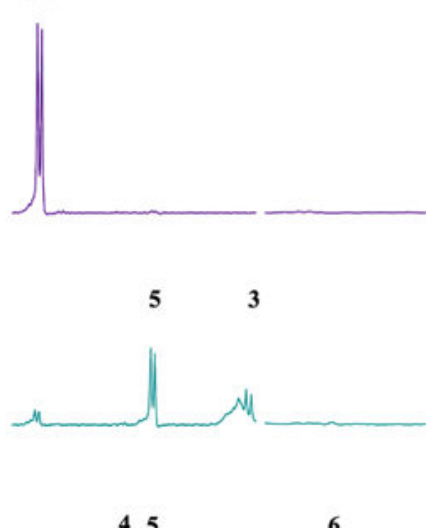

Figure 2.

In situ ${ }^{1} \mathrm{H}$ NMR analysis over time upon treatment of $\mathbf{2}$ with TFA (a) and CPA $\mathbf{F}$ (b). 


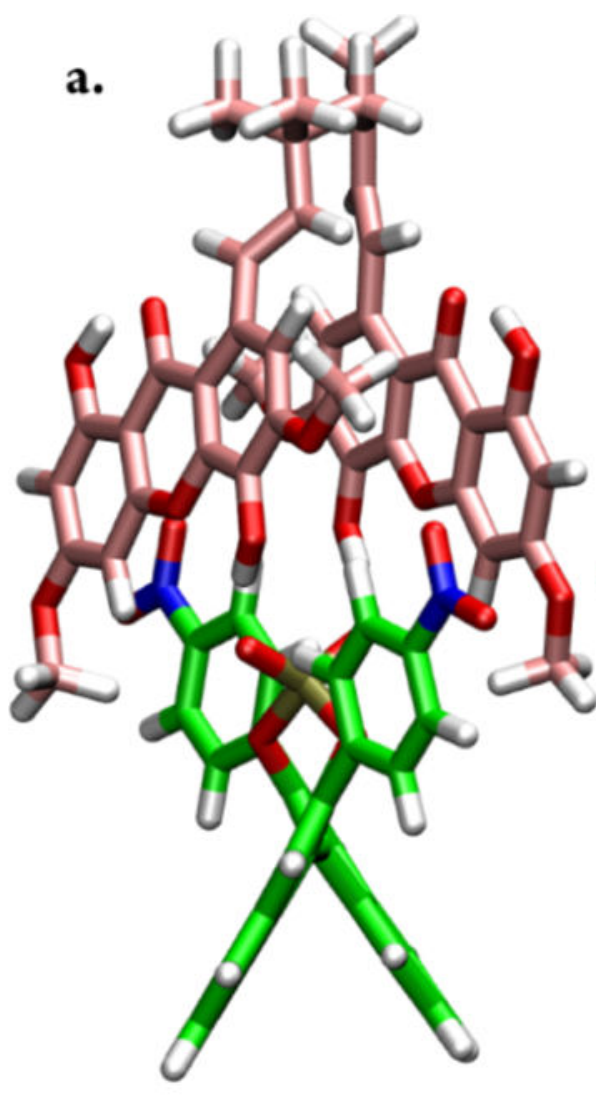

c.

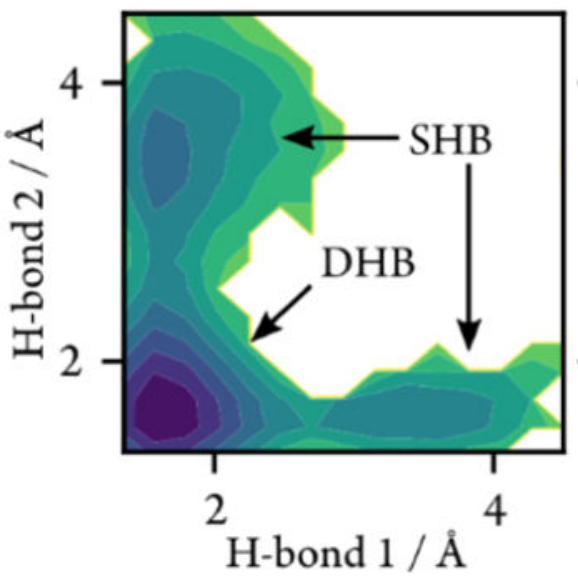

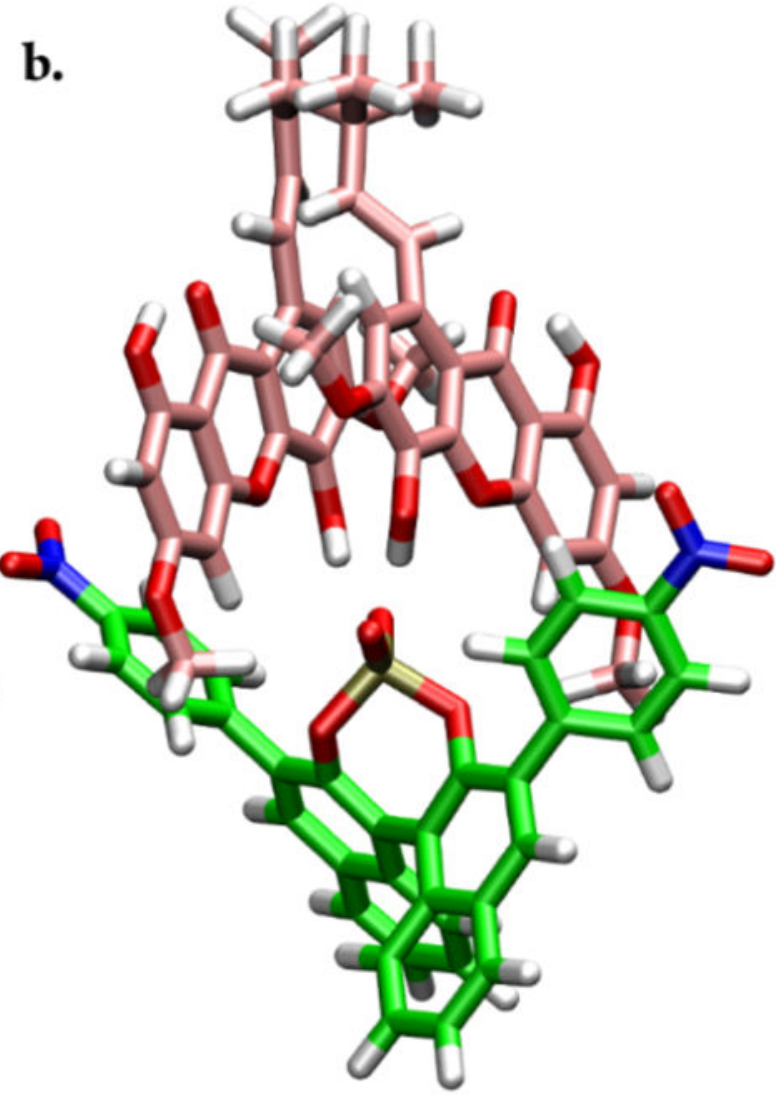

d.

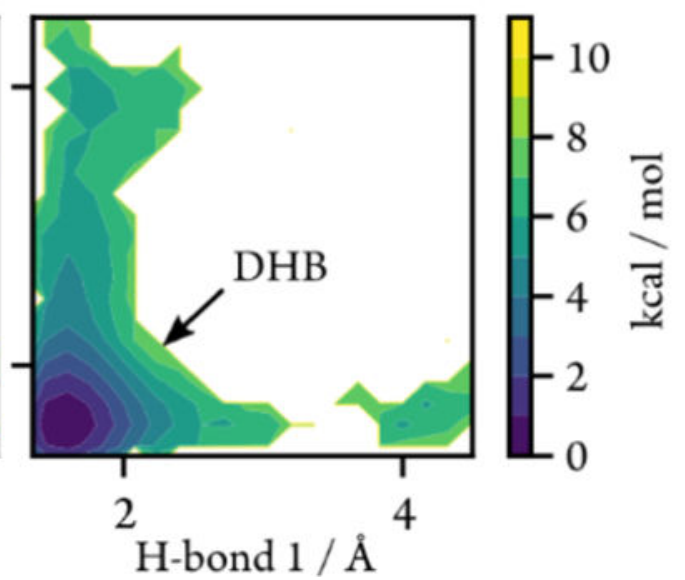

Figure 3.

Representative configurations of TS-1 (pink) doubly hydrogen bonding with CPA F (green). DFT-optimized (a) TS-1 - CPA F (0 kcal/mol) and (b) ent-TS-1-CPA F $(7 \mathrm{kcal} / \mathrm{mol})$. Free energy surface cuts at $T=298 \mathrm{~K}$ for (c) TS-1-CPA F simulation shows free energy minima with single hydrogen bonds (SHB) and DHB. (d) ent-TS-1-CPA F simulation reveals a single DHB minimum only. 

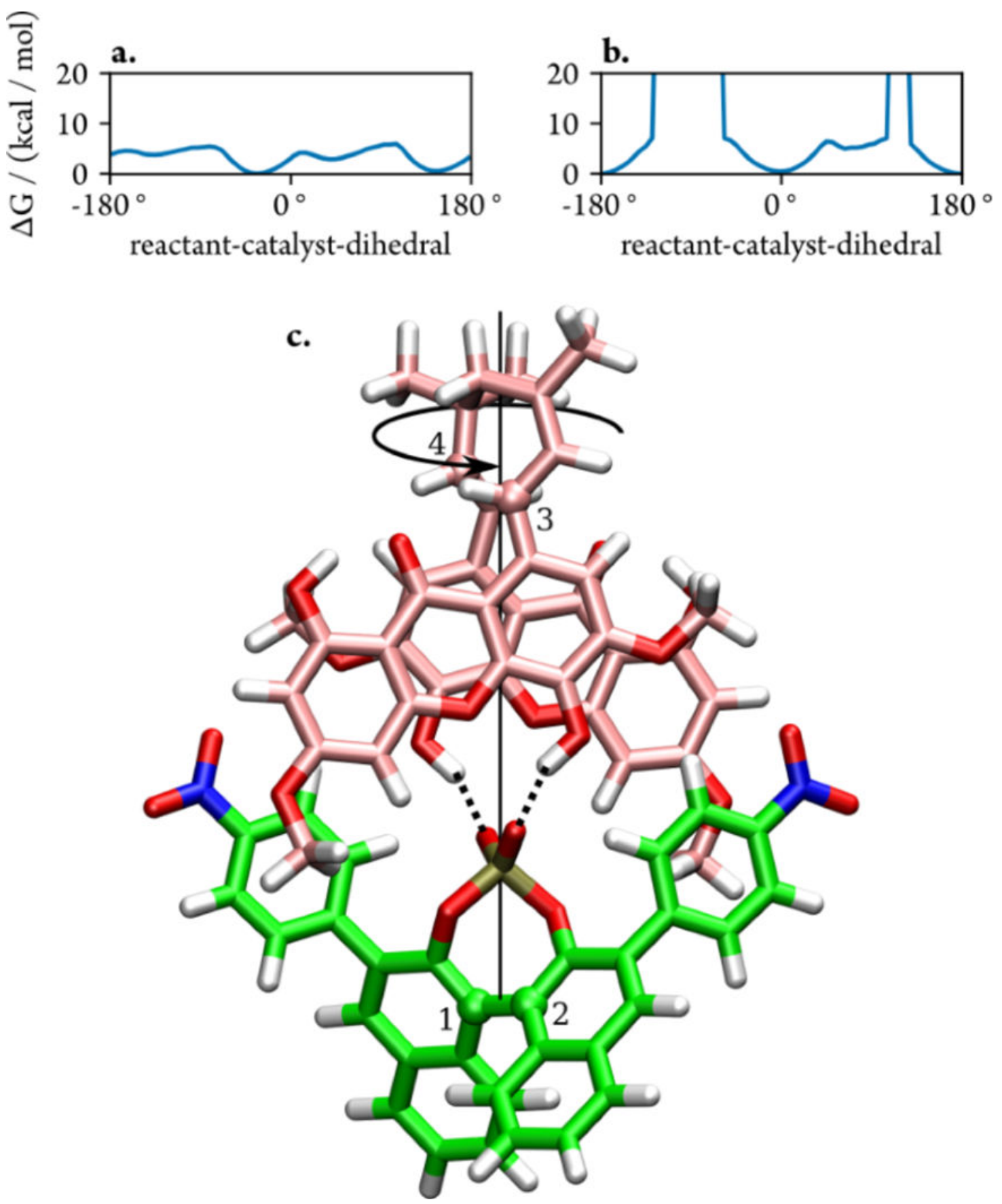

Figure 4.

$\left(a\right.$ and $b$ ) Free energy surfaces as a function of intermolecular dihedral angle, $\varphi_{\mathrm{D}}$, for TS-1 and ent-TS-1 with CPA F, respectively. Areas with a barrier of $>20 \mathrm{kcal} / \mathrm{mol}$ were not sampled. Greater rotation and flexibility is observed in TS-1 (c) as assessed by the free energy obtained from the intermolecular dihedral angle than in ent-TS-1, where free rotation relative to CPA $\mathbf{F}$ is inaccessible at the reaction temperature. 
a.

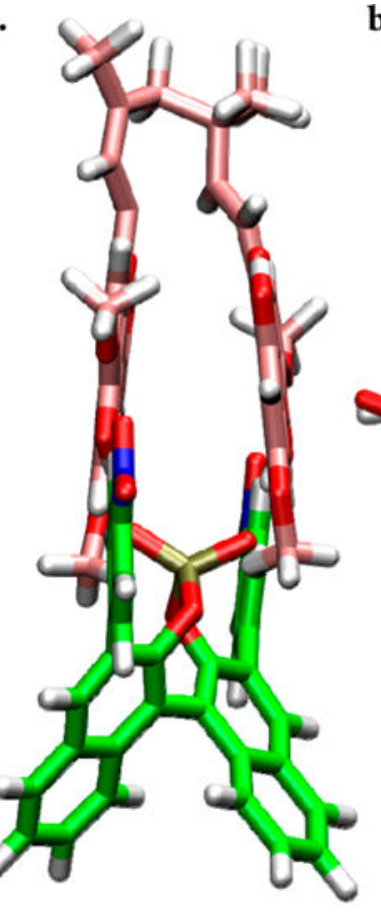

b.

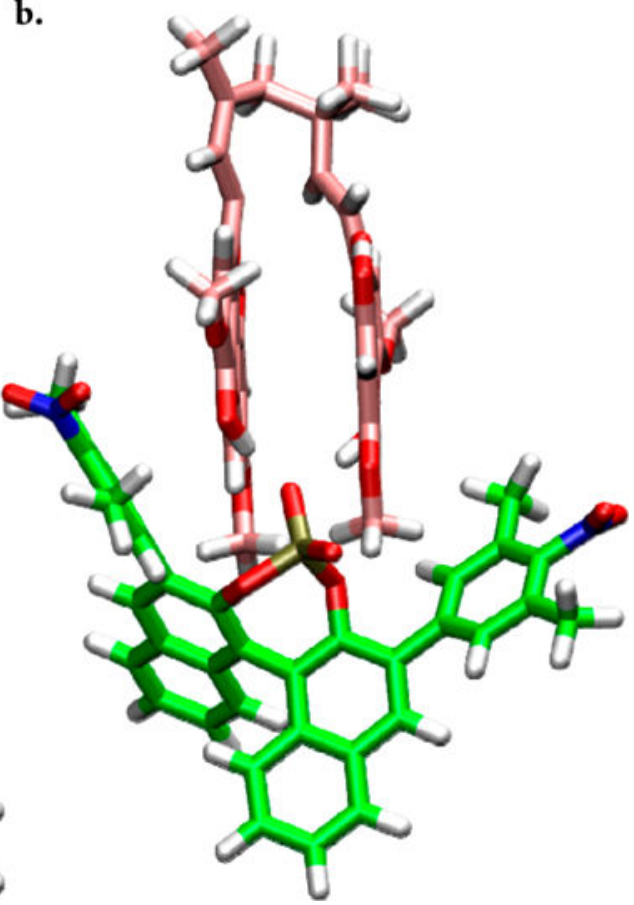

Figure 5.

Structural comparison of DFT-optimized (a) TS-1-CPA F and (b) TS-1-CPA G highlighting the altered complexation resulting from the sterically modified catalyst. 


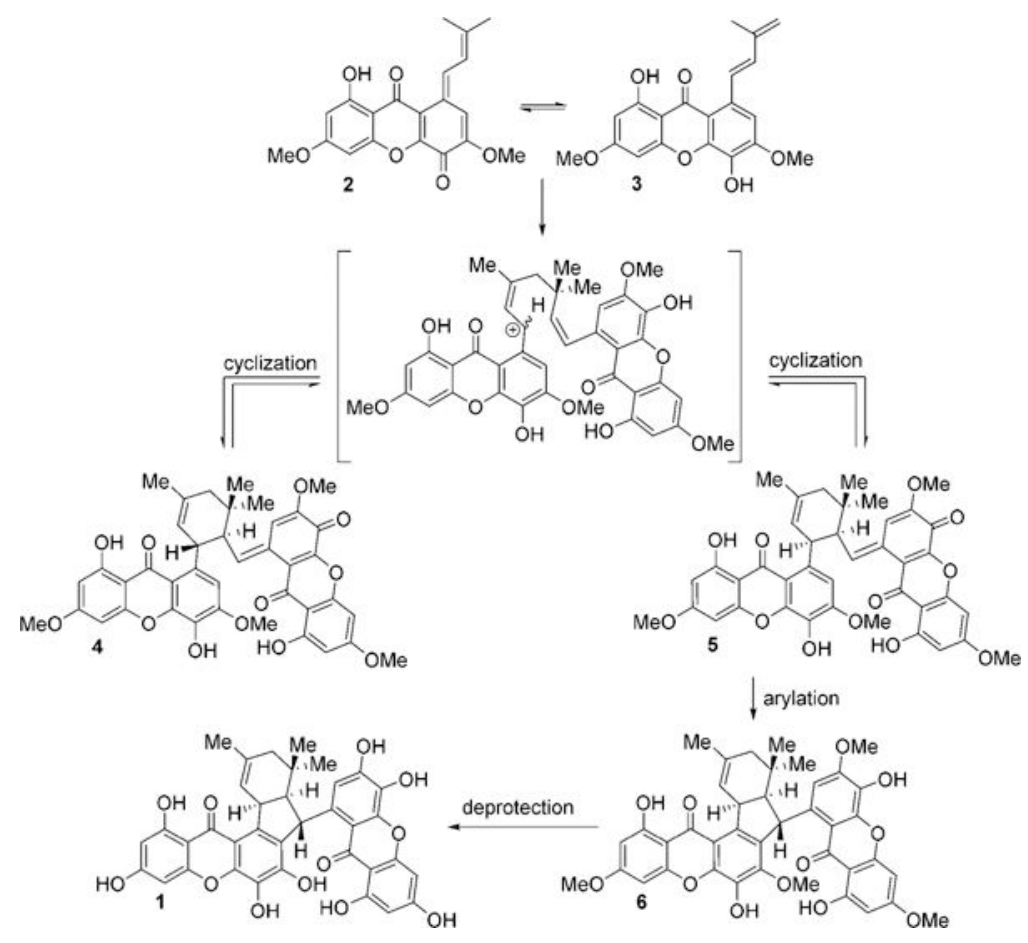

Scheme 1.

Brønsted Acid-Catalyzed Dimerization Cascade 
a. Sun and Coworkers:
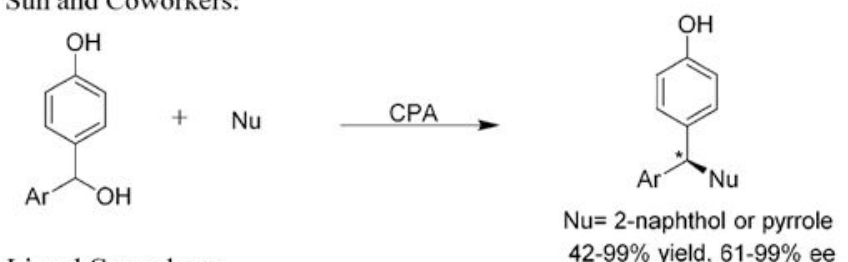

b. Li and Coworkers:

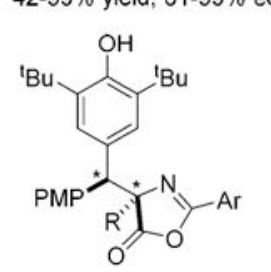

c. This work:

$68-85 \%$ yield, $88-98 \%$ ee

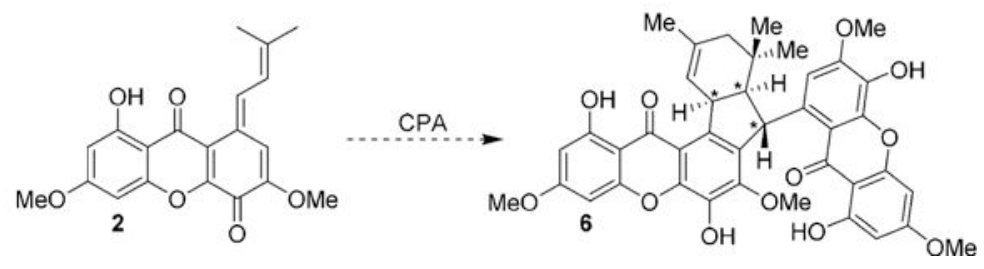

Scheme 2.

Representative CPA-Catalyzed Asymmetric Reactions of $p$-Quinone Methides 


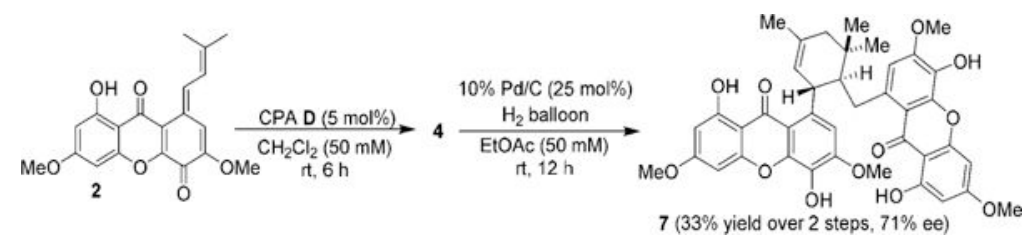

Scheme 3.

Reductive Trapping of anti-Cycloadduct 4 To Determine Enantioselectivity 
a.

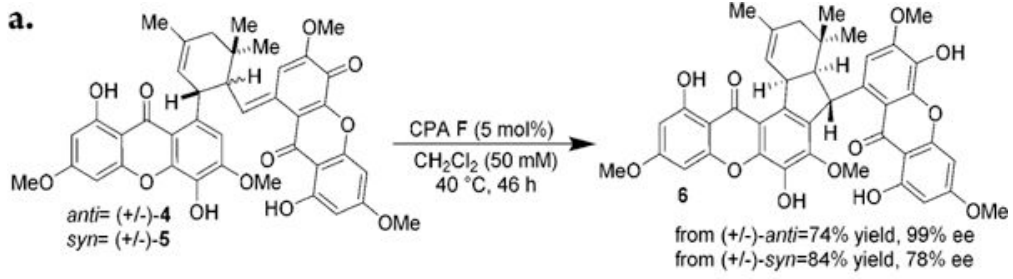

b.
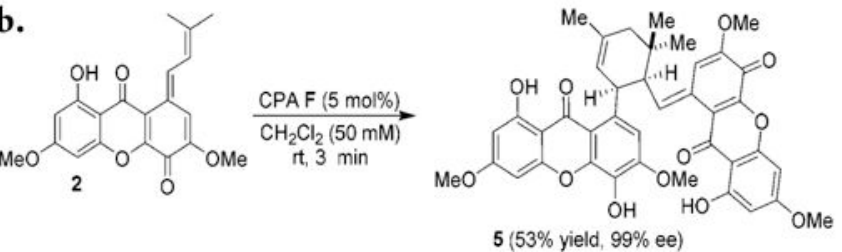

Scheme 4.

Enantioenrichment of ( \pm )-p-Quinone Methide Dimers 

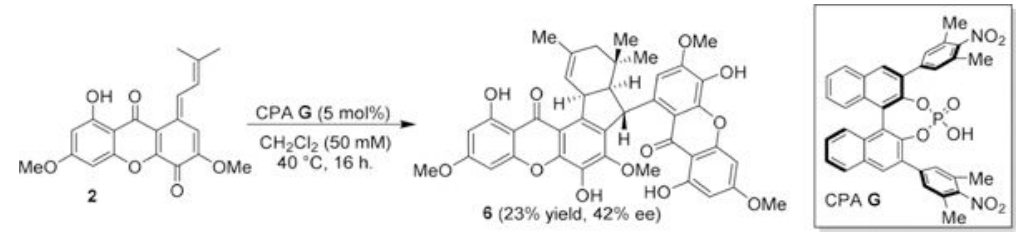

Scheme 5.

Disruption of Enantioselectivity Using a Modified CPA 
Table 1.

Assessment of BINOL-Derived CPAs

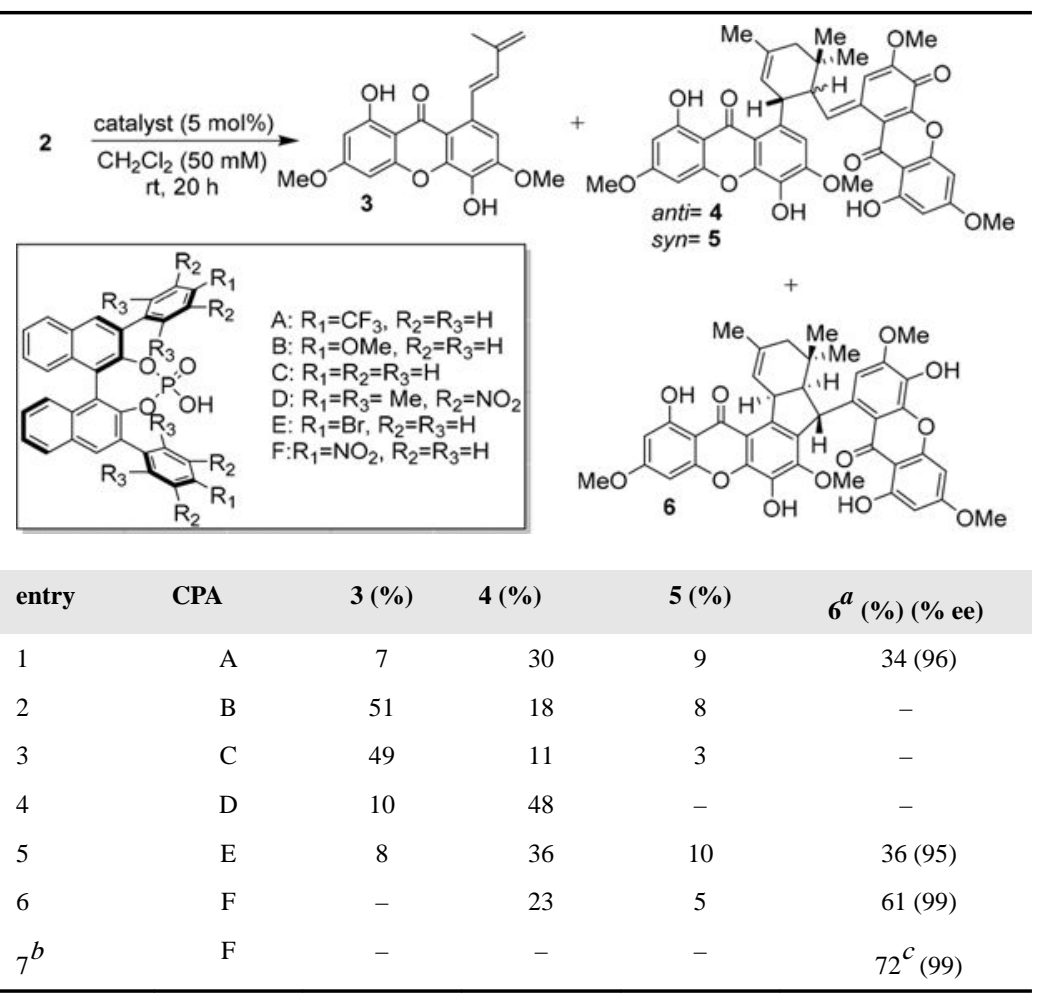

${ }^{a_{1}} \mathrm{H}$ NMR yields determined using 1,2,4,5-tetramethylbenzene as internal standard.

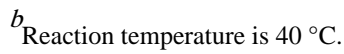

Isolated yield. 